\title{
Article
}

\section{Association between HOTAIR lncRNA Polymorphisms and Coronary Artery Disease Susceptibility}

\author{
In-Jai Kim ${ }^{1,+}$, Jeong-Yong Lee ${ }^{2,+}$, Hyeon-Woo Park ${ }^{2}$, Han-Sung Park ${ }^{2}$, Eun-Ju Ko ${ }^{2}$, Jung-Hoon Sung ${ }^{1}$ \\ and Nam-Keun Kim $2, *$ (D) \\ 1 CHA Bundang Medical Center, Department of Cardiology, CHA University, Seongnam 13496, Korea; \\ mdij24@chol.com (I.-J.K.); atropin5@cha.ac.kr (J.-H.S.) \\ 2 Department of Biomedical Science, College of Life Science, CHA University, Seongnam 13488, Korea; \\ smilee3625@naver.com (J.-Y.L.); aabb1114@naver.com (H.-W.P.); hahnsung@naver.com (H.-S.P.); \\ ejko05@naver.com (E.-J.K.) \\ * Correspondence: nkkim@cha.ac.kr; Tel.: +82-31-881-7137; Fax: +82-31-881-7249 \\ + Theses authors contributed equally to this work.
}

check for updates

Citation: Kim, I.-J.; Lee, J.-Y.; Park, H.-W.; Park, H.-S.; Ko, E.-J.; Sung, J.-H.; Kim, N.-K. Association between HOTAIR lncRNA

Polymorphisms and Coronary Artery Disease Susceptibility. J. Pers. Med. 2021, 11, 375. https://doi.org/ 10.3390/jpm11050375

Academic Editor:

Konstantinos Tziomalos

Received: 25 March 2021

Accepted: 26 April 2021

Published: 4 May 2021

Publisher's Note: MDPI stays neutral with regard to jurisdictional claims in published maps and institutional affiliations.

Copyright: (c) 2021 by the authors. Licensee MDPI, Basel, Switzerland. This article is an open access article distributed under the terms and conditions of the Creative Commons Attribution (CC BY) license (https:/ / creativecommons.org/licenses/by/ $4.0 /)$.

\begin{abstract}
Coronary artery disease (CAD), one of the most frequent causes of mortality, is the most common type of cardiovascular disease. This condition is characterized by the accumulation of plaques in the coronary artery, leading to blockage of blood flow to the heart. The main symptom of CAD is chest pain caused by blockage of the coronary artery and shortness of breath. HOX transcript antisense RNA gene (HOTAIR) is a long non-coding RNA which is well-known as an oncogene involved in various cancers, such as lung, breast, colorectal, and gastric cancer. We selected six single nucleotide polymorphisms, rs4759314 A>G, rs1899663 G>T, rs920778 T>C, rs7958904 G>C, rs12826786 $\mathrm{C}>\mathrm{T}$, and rs874945 C>T, for genotype frequency analysis and assessed the frequency of HOTAIR gene polymorphisms in $442 \mathrm{CAD}$ patients and 418 randomly selected control subjects. To analyze the differences between these two populations, we performed a Student's $t$-test, adjusted odds ratio (AOR), 95\% confidence intervals (CIs), and ANOVA analysis. According to our baseline characteristic analysis, control subjects and CAD patients were significantly different in hypertension and diabetes mellitus. We also found that the rs4759314 A>G, rs1899663 G>T, and rs12826786 C>T genotypes were strongly associated with CAD susceptibility (AA vs. AG+GG: AOR $=0.608,95 \% \mathrm{CI}=0.393-0.940$, $p=0.025$; GG vs. TT: $\mathrm{AOR}=2.276,95 \% \mathrm{CI}=1.125-4.607, p=0.022 ; \mathrm{CC}$ vs. CT+TT: AOR $=1.366$, $95 \% \mathrm{CI}=1.027-1.818, p=0.032$, respectively). Our data also demonstrated that the genotype of HOTAIR polymorphisms, genotype combination, and haplotype analysis affect disease occurrence. Moreover, these polymorphisms are linked to clinical factors that contribute to disease susceptibility. In conclusion, results from our study suggest that HOTAIR polymorphisms may be useful novel biomarkers for diagnosing CAD.
\end{abstract}

Keywords: single nucleotide variant; coronary artery disease; long non-coding RNA; HOX transcript antisense RNA

\section{Introduction}

The most common form of cardiovascular disease is coronary artery disease (CAD), in which a plaque builds up inside the coronary arteries, leading to a complete blockage of blood flow to the heart that can result in a heart attack and chest pain (angina). CAD is characterized by atherosclerosis in the epicardial coronary arteries. According to the World Health Organization, CAD is one of the most common causes of mortality [1], with classic risk factors that include age, gender, hypertension (HTN), diabetes mellitus (DM), and smoking [2,3].

Long non-coding RNAs (lncRNA) are known as a non-transcript RNA and characterized by a length greater than 200 nucleotides. The functions of lncRNA are not fully understood, but studies have suggested their involvement in regulating gene expression 
and post-transcriptional modification [4]. Moreover, lncRNA has been shown to play a role in various diseases, especially cancer and cardiovascular diseases, including myocardial infarction (MI), atrial fibrillation, and CAD [5-12].

Homeobox (HOX) transcript antisense RNA (HOTAIR) is a lncRNA located on chromosome 12q13.13 that is encoded in the HOXC gene cluster [13], which consists of $6232 \mathrm{nu}-$ cleotides [9]. HOTAIR activates HOXD gene expression by decreasing the tri-methylation of histone H3K27 [9] and recruiting the polycomb repressive complex (PRC2). HOTAIR also regulates histone $\mathrm{H} 3 \mathrm{~K} 4$, thus promoting interactions with lysine-specific histone demethylase 1A (LSD1) [9]. HOTAIR has been reported to be a key regulator of cancer, including colorectal, prostate, gastric, and ovarian cancer $[13,14]$. Finally, HOTAIR overexpression suppressed cell viability and upregulated cell apoptosis by oxidized low-density lipoprotein (LDL) [15].

HOTAIR is frequently reported as an oncogene; however, studies have also associated it with cardiovascular diseases, such as CAD and heart failure. Previous reports demonstrated that HOTAIR acts as a negative regulator of MI in mice by absolving miR519d-3p [16]. Moreover, HOTAIR was reported as an antagonist of cardiovascular disease that protects against hypoxia exposure or acute myocardial ischemia by absolving miR-1 and miR-125 to inhibit apoptosis and regulate downstream genes [17].

Single nucleotide polymorphisms (SNPs) are associated with many disease occurrences [18-20], and HOTAIR SNPs have been associated with recurrent implant failure and various cancers. However, the association between HOTAIR SNPs and CAD remains poorly understood. In this study, we analyzed the differences between CAD patients and healthy controls and found that the HOTAIR rs4759314 A>G, rs1899663 C>T, and rs12826786 C>T polymorphisms are genetically associated with the prevalence of $\mathrm{CAD}$.

\section{Materials and Methods}

\subsection{Study Population}

In the South Korean provinces of Seoul and Gyeonggi-do, study subjects were recruited between 2006 and 2015 from the Department of Cardiology at the CHA Bundang Medical Center in Seongnam, South Korea. The study was approved by the Institutional Review Board of CHA Bundang Medical Center. (IRB number: 2013-10-114) In total, 442 patients diagnosed with CAD were referred from the Department of Cardiology, and all patients who presented with stable CAD or acute coronary syndromes (including unstable angina with or without ST-segment elevation) and at least one coronary lesion with $>50 \%$ stenosis in a vessel with a diameter of $2.25-4.00 \mathrm{~mm}$ were screened for eligibility. No restrictions were placed on the total number of treated lesions, which vessels were treated, lesion length, or the number of stents implanted. Exclusion criteria included a history of acute myocardial infarction and life expectancy $<1$ year. All patients underwent coronary angiography and electrocardiography. Diagnoses were made by coronary angiography and confirmed by at least one independent experienced cardiologist.

During the same time period, 418 gender- and age-matched control subjects were selected from patients who visited the Department of Cardiology for health examinations, including biochemical testing, electrocardiograms, and coronary computed tomography scans. The control group was subjected to the same exclusion criteria as the CAD group, with the addition of reported recent history of anginal symptoms. Hypertension was defined as a systolic pressure $>140 \mathrm{mmHg}$ and a diastolic pressure $>90 \mathrm{mmHg}$ on more than one occasion and included patients who were treated with hypertensive medications during the study period. DM was defined as a fasting plasma glucose level $>126 \mathrm{mg} / \mathrm{dL}$ $(7.0 \mathrm{mmol} / \mathrm{L})$ and included patients taking diabetic medications. Smoking referred to patients who smoked at the time of the study. Hyperlipidemia was defined as a high fasting serum total cholesterol level ( $\geq 240 \mathrm{mg} / \mathrm{dL}$ ) or a history of anti-hyperlipidemic treatment. 


\subsection{Genetic Analyses}

Genomic DNA was extracted from whole blood samples using the G-DEX II Genomic DNA Extraction kit (Intron Biotechnology Inc., Seongnam, Korea). DNA was diluted to $100 \mathrm{ng} / \mu \mathrm{L}$ with $1 \times$ Tris-EDTA (TE) buffer, and then $1 \mu \mathrm{L}$ of each sample was used to amplify the polymorphisms.

All PCR experiments were performed using the AccuPower HotStart PCR PreMix (Bioneer Corporation, Daejeon, Korea). For the genotyping analysis, rs7958904, rs920778, and rs12826786 were analyzed using a Taqman genotyping assay (Applied Biosystems, Foster City, CA, USA), whereas rs1899663 and rs4759314 genotyping was performed using polymerase chain reaction-restriction fragment length polymorphism (PCR-RFLP) analysis. The following primer sets were used in this study: rs1899663, 5'- TTT TCC AGT TGA GGA GGG TGG A -3' (forward primer) and 5'- CTA ATG GCA AGG GAA GGG AAG G -3' (reverse primer); rs4759314, 5'- ACC CAA AAC CAT TTC CTG AGA G -3' (forward primer) and 5'- TTC AGG TTT TAT TAA CTT GCA TCA GC -3' (reverse primer). HphI and $A l u \mathrm{I}$ restriction enzymes were used in this study (Table S1). Taqman probes were obtained directly from Applied Biosystems, and genotyping was performed according to the manufacturer's protocols.

\subsection{Statistical Analysis}

To estimate the relative CAD risk of the various genotypes, the odds ratio (OR) and 95\% CI were calculated. The statistical significance of differences between the CAD and control groups was determined by the Student's $t$-test for continuous variables and the $\chi 2$ test for categorical variables. For multivariate analyses, logistic regression analysis was performed to adjust for possible confounders, including age, gender, hypertension, DM, hyperlipidemia, and smoking. A $p$-value less than 0.05 was considered to be a statistically significant difference. Multiple hypothesis testing was performed using the BenjaminiHochberg method to control the false discovery rate (FDR) in the logistic regression analysis. Calculating the FDR enabled us to address problems associated with multiple comparisons, and FDR provides a measure of the expected proportion of false positives among data. Analyses were performed using GraphPad Prism 4.0 (GraphPad Software, Inc., San Diego, CA, USA), StatsDirect statistical software Version 2.4.4 (StatsDirect Ltd., Altrincham, UK), and MedCalc (Version 7.4 for Windows; MedCalc, Ostend, Belgium).

The multifactor dimensionality reduction (MDR) method was described previously [21,22]. Briefly, the MDR is comprised of two steps. First, the best combination of multifactors is selected, and then the genotype combinations are classified into high- and low-risk groups [22]. Interaction analyses were performed using the open-source MDR software package (v.2.0) available from www.epistasis.org. (released in 2008) Using these MDR analyses, all possible allelic combinations were identified for gene-gene interactions. HAPSTAT software was used to estimate the frequencies of allelic combinations for the polymorphisms selected by the MDR analysis with strong synergistic effects. HAPSTAT enables testing of haplotype (or allelic combination) effects by maximizing the likelihood (from the observed data) of properly accounting for phase uncertainty and study design. Current versions of the HAPSTAT software (v.3.0) are available from www.bios.unc.edu/ lin/hapstat/ (University of North Carolina, Chapel Hill, NC, USA; released in 2008).

\section{Results}

\subsection{Characteristics of the Study Population}

The baseline characteristics of the CAD and control groups are summarized in Table 1. No significant differences in age and gender distribution were observed between the CAD and control groups, indicating that our frequency matching on age and gender was satisfactory. The average body mass index (BMI) of CAD patients was significantly higher than that of the control group $(p=0.0008)$. In addition, CAD patients exhibited significantly lower serum total cholesterol $(p<0.001)$ and high-density lipoprotein (HDL) cholesterol $(p=0.001)$ levels compared with the control group. However, no difference was observed 
in the level of serum LDL cholesterol $(p=0.896)$ and triglyceride (TG) $(p=0.871)$ between the two groups. For disease history, 114 (26.1\%) patients had a history of DM, which was significantly higher compared with controls $(p<0.0001)$. A significant difference was also observed in the hypertension history between CAD patients and controls $(p=0.0007)$. Nevertheless, no significant differences were found with smoking $(p=0.539)$ or hyperlipidemia history $(p=0.263)$ between CAD patients and the control group.

Table 1. Baseline characteristics of control subjects and coronary artery disease patients.

\begin{tabular}{|c|c|c|c|}
\hline Characteristics & $\begin{array}{l}\text { Controls } \\
(n=418)\end{array}$ & $\begin{array}{c}\text { CAD } \\
(n=442)\end{array}$ & $P^{a}$ \\
\hline Age (years, mean \pm SD) & $61.43 \pm 11.15$ & $61.35 \pm 11.50$ & 0.521 \\
\hline Male (\%) & $172(41.1)$ & $186(42.1)$ & 0.084 \\
\hline Hypertension (\%) & $169(40.4)$ & $228(52.2)$ & 0.0007 \\
\hline Systolic BP $(\mathrm{mmHg}$, mean $\pm \mathrm{SD})$ & $131.47 \pm 17.21$ & $127.75 \pm 20.58$ & $<0.001$ \\
\hline Diastolic BP (mmHg, mean $\pm \mathrm{SD})$ & $80.33 \pm 11.84$ & $78.77 \pm 12.81$ & 0.101 \\
\hline Diabetes mellitus (\%) & $59(14.1)$ & $114(26.1)$ & $<0.0001$ \\
\hline Fasting blood sugar $(\mathrm{mg} / \mathrm{dL}$, mean $\pm \mathrm{SD})$ & $113.93 \pm 37.51$ & $141.02 \pm 62.84$ & $<0.001$ \\
\hline Hemoglobin A1c $(\%$, mean \pm SD $)$ & $6.24 \pm 1.44$ & $6.42 \pm 1.51$ & 0.568 \\
\hline Hyperlipidemia $(n, \%)$ & $100(23.9)$ & $121(27.5)$ & 0.263 \\
\hline $\mathrm{BMI} \geq 25 \mathrm{~kg} / \mathrm{m}^{2}, n(\%)$ & $103(38.3)$ & $224(51.6)$ & 0.0008 \\
\hline Total cholesterol $(\mathrm{mg} / \mathrm{dL}$, mean $\pm \mathrm{SD})$ & $192.15 \pm 37.23$ & $187.37 \pm 47.04$ & $<0.001$ \\
\hline Triglyceride $(\mathrm{mg} / \mathrm{dL}$, mean $\pm \mathrm{SD})$ & $146.92 \pm 91.26$ & $158.21 \pm 90.55$ & 0.871 \\
\hline LDL cholesterol $(\mathrm{mg} / \mathrm{dL}$, mean $\pm \mathrm{SD})$ & $116.16 \pm 41.36$ & $112.49 \pm 41.07$ & 0.896 \\
\hline HDL cholesterol (mg/dL, mean $\pm \mathrm{SD})$ & $46.37 \pm 13.77$ & $43.68 \pm 11.21$ & 0.001 \\
\hline Smokers (\%) & $138(33.0)$ & $135(30.8)$ & 0.539 \\
\hline Folate $(\mathrm{nmol} / \mathrm{L}$, mean $\pm \mathrm{SD})$ & $8.77 \pm 6.26$ & $8.78 \pm 9.76$ & $<0.001$ \\
\hline Vitamin B12 (pg/mL, mean \pm SD) & $686.11 \pm 268.65$ & $693.47 \pm 306.38$ & 0.102 \\
\hline Homocysteine $(\mu \mathrm{mol} / \mathrm{L}$, mean $\pm \mathrm{SD})$ & $9.84 \pm 4.14$ & $9.65 \pm 4.67$ & 0.014 \\
\hline
\end{tabular}

Note: CAD, coronary artery disease; SD, standard deviation; HDL, high-density lipoprotein; LDL, low-density lipoprotein. $P^{a}$ was calculated using the Mann-Whitney test for continuous variables and Chi-square test for categorical variables.

\section{2. rs4759314 A>G, rs1899663 G>T, and rs12826786 C>T Polymorphisms Are Significantly Correlated with $C A D$}

In this study, we investigated the distribution of the HOTAIR rs4759314 A>G, rs1899663 G>T, rs920778 T>C, rs7958904 G>C, rs12826786 C>T, and rs874945 C>T polymorphisms in CAD patients and the control group (Table 2). The adjusted odds ratio (AOR) from logistic regression analyses with respect to age, gender, hypertension, and DM was calculated. The frequency of each genotype in the control group was consistent with the Hardy-Weinberg equilibrium. Our analysis revealed that several polymorphisms differed significantly between the CAD and control groups, including the rs4759314 A>G (AA vs. AG: crude odds ratio COR, 0.642, 95\% CI, 0.414-0.998; AOR, 0.620, 95\% CI, 0.395-0.973), rs1899663 G>T (GG vs. TT: COR, 2.276, 95\% CI, 1.125-4.607; GG+GT vs. TT: COR, 2.130, 95\% CI, 1.046-4.340), and rs12826786 C> T polymorphisms (CC vs. CT: COR, 1.355, 95\% CI, 1.017-1.805, AOR, 1.347, 95\% CI, 1.005-1.805). However, the rs920778 T>C, rs7958904 G>C, and rs874945 C>T polymorphisms were not significantly different between the CAD and control groups (Table 2).

\subsection{Relationship between Metabolic Syndrome and Genotype Frequencies}

We also conducted a logistic regression analysis to investigate the relationship between metabolic syndrome and the genotype frequency of each polymorphism, with each polymorphism adjusted by age, gender, hypertension, and DM. A comparison between the non-metabolic syndrome control subjects and CAD patients revealed that the rs1899663 $\mathrm{G}>\mathrm{T}$ and $\mathrm{rs} 12826786 \mathrm{C}>\mathrm{T}$ polymorphisms were related to CAD occurrence (rs1899663, GG vs. GT, AOR, 1.577, 95\% CI, 1.054-2.361; CC vs. CT: AOR, 1.577, 95\% CI, 1.054-2.361). Only the rs1899663 G>T genotype was found to be associated with CAD (rs1899663, GG vs. TT, AOR, 3.522, 95\% CI, 1.162-10.676) when non-metabolic syndrome controls and metabolic syndrome patients were compared. However, rs12826786C >T was not significantly as- 
sociated with CAD prevalence in non-metabolic syndrome control subjects or metabolic syndrome patients (Table 3).

Table 2. Comparison of genotype frequencies and AOR values of HOTAIR polymorphisms between the CAD and control subjects.

\begin{tabular}{|c|c|c|c|c|c|c|}
\hline Genotypes & Controls $(n=418)$ & CAD $(n=442)$ & COR $(95 \% \mathrm{CI})$ & $p$ & AOR $(95 \% \mathrm{CI})$ & $p$ \\
\hline \multicolumn{7}{|l|}{ rs4759314 A>G } \\
\hline AA & $362(86.6)$ & $404(76.2)$ & 1.000 (reference) & & & \\
\hline AG & $53(12.7)$ & $38(7.2)$ & $0.642(0.414-0.998)$ & 0.049 & $0.620(0.395-0.973)$ & 0.037 \\
\hline GG & $3(0.7)$ & $0(0.0)$ & $\mathrm{N} / \mathrm{A}$ & N/A & $\mathrm{N} / \mathrm{A}$ & $\mathrm{N} / \mathrm{A}$ \\
\hline $\begin{array}{l}\text { Dominant (AA vs. } \\
\qquad A G+G G)\end{array}$ & & & $0.608(0.393-0.940)$ & 0.025 & $0.586(0.375-0.916)$ & 0.019 \\
\hline $\begin{array}{l}\text { Recessive (AA+AG vs. } \\
\text { GG) }\end{array}$ & & & $\mathrm{N} / \mathrm{A}$ & $\mathrm{N} / \mathrm{A}$ & $\mathrm{N} / \mathrm{A}$ & $\mathrm{N} / \mathrm{A}$ \\
\hline HWE-P & 0.494 & 0.345 & & & & \\
\hline \multicolumn{7}{|l|}{ rs1899663 G>T } \\
\hline GG & $270(64.6)$ & $257(48.5)$ & 1.000 (reference) & & & \\
\hline GT & $136(32.5)$ & $159(30.0)$ & $1.228(0.923-1.634)$ & 0.158 & $1.245(0.929-1.667)$ & 0.142 \\
\hline $\mathrm{TT}$ & $12(2.9)$ & $26(4.9)$ & $2.276(1.125-4.607)$ & 0.022 & $2.284(1.110-4.700)$ & 0.025 \\
\hline $\begin{array}{c}\text { Dominant (GG vs. } \\
\text { GT+TT) }\end{array}$ & & & $1.313(0.997-1.730)$ & 0.053 & $1.336(1.008-1.772)$ & 0.044 \\
\hline Recessive (GG+GT vs. TT) & & & $2.115(1.053-4.248)$ & 0.035 & $2.140(1.052-4.357)$ & 0.036 \\
\hline HWE-P & 0.295 & 0.830 & & & & \\
\hline \multicolumn{7}{|l|}{ rs920778 T>C } \\
\hline $\mathrm{TT}$ & $243(58.1)$ & $231(43.6)$ & 1.000 (reference) & & & \\
\hline $\mathrm{TC}$ & $151(36.1)$ & $184(34.7)$ & $1.282(0.968-1.697)$ & 0.083 & $1.261(0.946-1.680)$ & 0.114 \\
\hline $\mathrm{CC}$ & $24(5.7)$ & $27(5.1)$ & $1.183(0.664-2.111)$ & 0.568 & $1.167(0.646-2.110)$ & 0.608 \\
\hline $\begin{array}{c}\text { Dominant (TT vs. } \\
\text { TC+CC) }\end{array}$ & & & $1.268(0.969-1.661)$ & 0.084 & $1.251(0.950-1.647)$ & 0.111 \\
\hline Recessive (TT+TC vs. CC) & & & $1.068(0.606-1.883)$ & 0.820 & $1.062(0.596-1.892)$ & 0.839 \\
\hline HWE-P & 0.932 & 0.223 & & & & \\
\hline \multicolumn{7}{|l|}{ rs7958904 G>C } \\
\hline GG & $250(59.8)$ & $246(46.4)$ & 1.000 (reference) & & & \\
\hline GC & $139(33.3)$ & 167 (31.5) & $1.221(0.918-1.625)$ & 0.171 & $1.187(0.886-1.591)$ & 0.25 \\
\hline $\mathrm{CC}$ & $29(6.9)$ & $29(5.5)$ & $1.016(0.590-1.751)$ & 0.954 & $0.980(0.561-1.709)$ & 0.942 \\
\hline $\begin{array}{l}\text { Dominant (GG vs. } \\
\text { GC+CC) }\end{array}$ & & & $1.186(0.904-1.555)$ & 0.218 & $1.153(0.874-1.520)$ & 0.315 \\
\hline $\begin{array}{c}\text { Recessive (GG+GC vs. } \\
\text { CC) }\end{array}$ & & & $0.942(0.553-1.605)$ & 0.826 & $0.917(0.533-1.577)$ & 0.753 \\
\hline HWE-P & 0.116 & 0.927 & & & & \\
\hline \multicolumn{7}{|l|}{ rs12826786 C>T } \\
\hline $\mathrm{CC}$ & $282(67.5)$ & $266(50.2)$ & 1.000 (reference) & & & \\
\hline $\mathrm{CT}$ & $126(30.1)$ & $161(30.4)$ & $1.355(1.017-1.805)$ & 0.038 & $1.338(0.998-1.794)$ & 0.052 \\
\hline TT & $10(2.4)$ & $15(2.8)$ & $1.590(0.702-3.602)$ & 0.266 & $1.589(0.686-3.679)$ & 0.28 \\
\hline $\begin{array}{c}\text { Dominant (CC vs. } \\
\text { CT+TT) }\end{array}$ & & & $1.372(1.037-1.814)$ & 0.027 & $1.360(1.022-1.810)$ & 0.035 \\
\hline Recessive $(\mathrm{CC}+\mathrm{CT}$ vs. TT) & & & $1.433(0.637-3.227)$ & 0.385 & $1.472(0.643-3.369)$ & 0.36 \\
\hline HWE-P & 0.351 & 0.114 & & & & \\
\hline \multicolumn{7}{|l|}{ rs874945 C>T } \\
\hline $\mathrm{CC}$ & $267(63.9)$ & $258(48.7)$ & 1.000 (reference) & & & \\
\hline $\mathrm{CT}$ & $133(31.8)$ & $164(30.9)$ & $1.276(0.959-1.698)$ & 0.094 & $1.249(0.932-1.673)$ & 0.137 \\
\hline TT & $18(4.3)$ & $20(3.8)$ & $1.150(0.595-2.223)$ & 0.678 & $1.131(0.573-2.230)$ & 0.723 \\
\hline $\begin{array}{c}\text { Dominant (CC vs. } \\
\text { CT+TT) }\end{array}$ & & & $1.261(0.958-1.660)$ & 0.098 & $1.246(0.940-1.651)$ & 0.126 \\
\hline Recessive (CC+CT vs. TT) & & & $1.053(0.549-2.020)$ & 0.876 & $1.078(0.555-2.095)$ & 0.824 \\
\hline HWE-P & 0.781 & 0.343 & & & & \\
\hline
\end{tabular}

Note: AOR was adjusted by age, gender, hypertension, diabetes mellitus, and hyperlipidemia. COR = crude odds ratio; AOR = adjusted odds ratio; $95 \% \mathrm{CI}=95 \%$ confidence interval; $\mathrm{HWE}=$ Hardy-Weinberg equilibrium. 
Table 3. Comparison of genotype frequencies and AOR values of HOTAIR polymorphisms between metabolic syndromes.

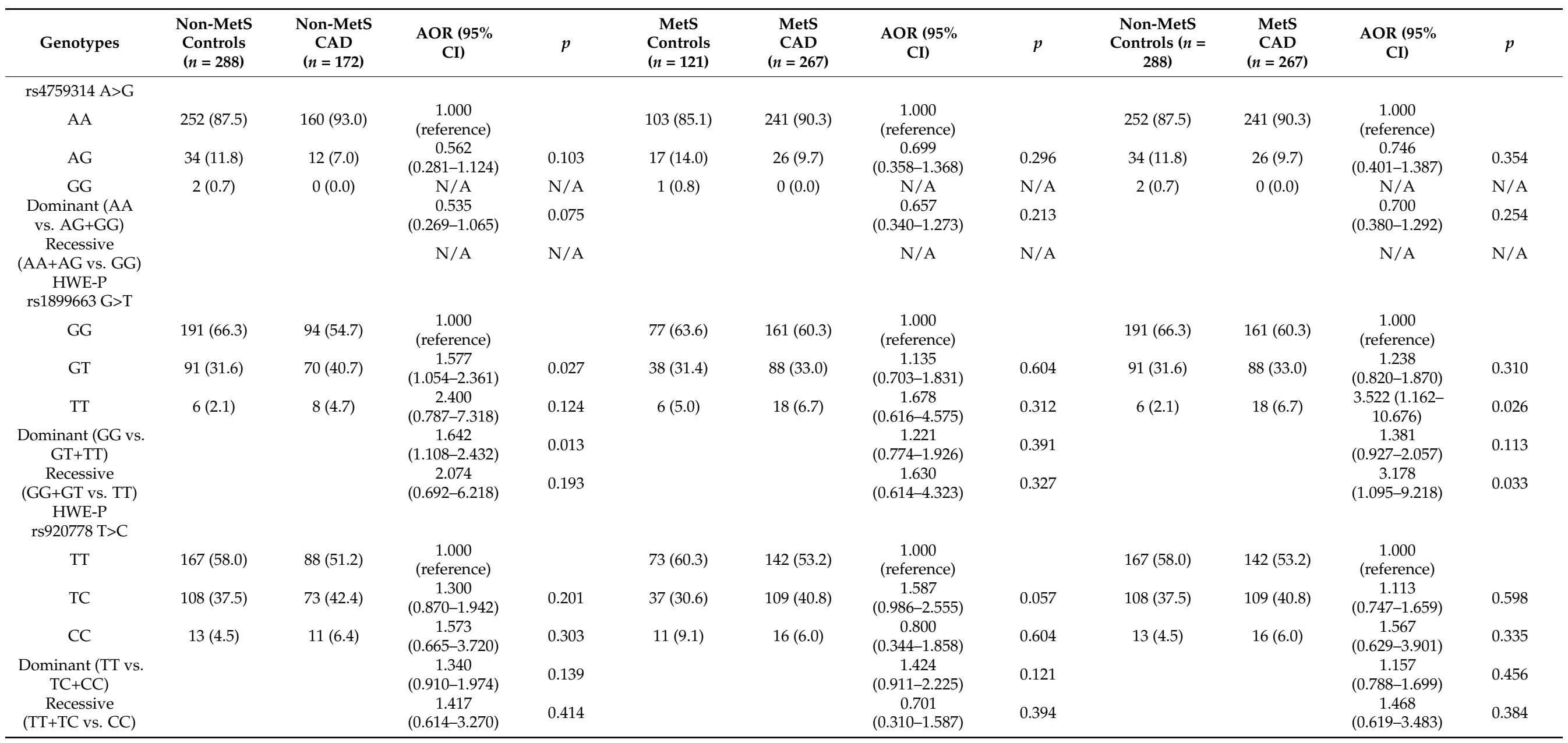


Table 3. Cont.

\begin{tabular}{|c|c|c|c|c|c|c|c|c|c|c|c|c|}
\hline Genotypes & $\begin{array}{l}\text { Non-MetS } \\
\text { Controls } \\
(n=288)\end{array}$ & $\begin{array}{c}\text { Non-MetS } \\
\text { CAD } \\
(n=172)\end{array}$ & $\begin{array}{l}\text { AOR (95\% } \\
\text { CI) }\end{array}$ & $p$ & $\begin{array}{c}\text { MetS } \\
\text { Controls } \\
(n=121) \\
\end{array}$ & $\begin{array}{c}\text { MetS } \\
\text { CAD } \\
(n=267) \\
\end{array}$ & $\begin{array}{c}\text { AOR }(95 \% \\
\text { CI) }\end{array}$ & $p$ & $\begin{array}{c}\text { Non-MetS } \\
\text { Controls }(n= \\
288)\end{array}$ & $\begin{array}{c}\text { MetS } \\
\text { CAD } \\
(n=267)\end{array}$ & $\begin{array}{l}\text { AOR }(95 \% \\
\text { CI) }\end{array}$ & $p$ \\
\hline \multicolumn{13}{|l|}{$\begin{array}{c}\text { HWE-P } \\
\text { rs7958904 G>C }\end{array}$} \\
\hline GG & $173(60.1)$ & 89 (51.7) & $\begin{array}{c}1.000 \\
\text { (reference) }\end{array}$ & & 74 (61.2) & $156(58.4)$ & $\begin{array}{c}1.000 \\
\text { (reference) }\end{array}$ & & $173(60.1)$ & $156(58.4)$ & $\begin{array}{c}1.000 \\
\text { (reference) }\end{array}$ & \\
\hline GC & 97 (33.7) & $73(42.4)$ & $\begin{array}{c}1.449 \\
(0.967-2.170)\end{array}$ & 0.072 & $36(29.8)$ & $92(34.5)$ & $\begin{array}{c}1.246 \\
(0.769-2.020)\end{array}$ & 0.372 & 97 (33.7) & $92(34.5)$ & $\begin{array}{c}0.975 \\
(0.646-1.472)\end{array}$ & 0.904 \\
\hline $\mathrm{CC}$ & $18(6.3)$ & $10(5.8)$ & $\begin{array}{c}0.986 \\
(0.423-2.296)\end{array}$ & 0.974 & $11(9.1)$ & $19(7.1)$ & $\begin{array}{c}0.864 \\
(0.384-1.947)\end{array}$ & 0.725 & $18(6.3)$ & $19(7.1)$ & $\begin{array}{c}1.040 \\
(0.469-2.304)\end{array}$ & 0.924 \\
\hline $\begin{array}{l}\text { Dominant (GG vs. } \\
\text { GC+CC) }\end{array}$ & & & $\begin{array}{c}1.395 \\
(0.946-2.056)\end{array}$ & 0.093 & & & $\begin{array}{c}1.164 \\
(0.743-1.822)\end{array}$ & 0.508 & & & $\begin{array}{c}0.994 \\
(0.674-1.466)\end{array}$ & 0.975 \\
\hline $\begin{array}{c}\text { Recessive } \\
\text { (GG+GC vs. CC) } \\
\text { HWE-P } \\
\text { rs12826786 C>T }\end{array}$ & & & $\begin{array}{c}0.870 \\
(0.387-1.956)\end{array}$ & 0.735 & & & $\begin{array}{c}0.814 \\
(0.368-1.799)\end{array}$ & 0.611 & & & $\begin{array}{c}1.093 \\
(0.508-2.350)\end{array}$ & 0.820 \\
\hline $\mathrm{CC}$ & 197 (68.4) & 99 (57.6) & $\begin{array}{c}1.000 \\
\text { (reference) }\end{array}$ & & 81 (66.9) & $166(62.2)$ & $\begin{array}{c}1.000 \\
\text { (reference) }\end{array}$ & & 197 (68.4) & $166(62.2)$ & $\begin{array}{c}1.000 \\
\text { (reference) }\end{array}$ & \\
\hline $\mathrm{CT}$ & $84(29.2)$ & $69(40.1)$ & $\begin{array}{c}1.633 \\
(1.089-2.450)\end{array}$ & 0.018 & 37 (30.6) & 90 (33.7) & $\begin{array}{c}1.211 \\
(0.754-1.946)\end{array}$ & 0.428 & $84(29.2)$ & $90(33.7)$ & $\begin{array}{c}1.283 \\
(0.847-1.944)\end{array}$ & 0.240 \\
\hline TT & $7(2.4)$ & $4(2.3)$ & $\begin{array}{c}1.117 \\
(0.310-4.025)\end{array}$ & 0.866 & $3(2.5)$ & $11(4.1)$ & $\begin{array}{c}1.965 \\
(0.513-7.526)\end{array}$ & 0.324 & $7(2.4)$ & $11(4.1)$ & $\begin{array}{c}1.506 \\
(0.483-4.696)\end{array}$ & 0.480 \\
\hline $\begin{array}{c}\text { Recessive } \\
\text { (CC+CT vs. TT) } \\
\text { HWE-P } \\
\text { rs874945 C>T }\end{array}$ & & & $\begin{array}{c}0.926 \\
(0.262-3.274)\end{array}$ & 0.905 & & & $\begin{array}{c}1.832 \\
(0.491-6.836)\end{array}$ & 0.367 & & & $\begin{array}{c}1.499 \\
(0.500-4.489)\end{array}$ & 0.470 \\
\hline $\mathrm{CC}$ & $185(64.2)$ & $98(57.0)$ & $\begin{array}{c}1.000 \\
\text { (reference) }\end{array}$ & & 78 (64.5) & 159 (59.6) & $\begin{array}{c}1.000 \\
\text { (reference) }\end{array}$ & & $185(64.2)$ & 159 (59.6) & $\begin{array}{c}1.000 \\
\text { (reference) }\end{array}$ & \\
\hline $\mathrm{CT}$ & 91 (31.6) & $68(39.5)$ & $\begin{array}{c}1.392 \\
(0.928-2.087)\end{array}$ & 0.11 & 37 (30.6) & $94(35.2)$ & $\begin{array}{c}1.296 \\
(0.805-2.086)\end{array}$ & 0.286 & 91 (31.6) & $94(35.2)$ & $\begin{array}{c}1.222 \\
(0.811-1.841)\end{array}$ & 0.339 \\
\hline TT & $12(4.2)$ & $6(3.5)$ & $\begin{array}{c}0.752 \\
(0.262-2.155)\end{array}$ & 0.596 & $6(5.0)$ & $14(5.2)$ & $\begin{array}{c}1.323 \\
(0.474-3.694)\end{array}$ & 0.593 & $12(4.2)$ & $14(5.2)$ & $\begin{array}{c}1.522 \\
(0.585-3.960)\end{array}$ & 0.389 \\
\hline $\begin{array}{c}\text { Dominant (CC vs. } \\
\text { CT+TT) }\end{array}$ & & & $\begin{array}{c}1.322 \\
(0.893-1.958)\end{array}$ & 0.164 & & & $\begin{array}{c}1.321 \\
(0.837-2.085)\end{array}$ & 0.233 & & & $\begin{array}{c}1.260 \\
(0.848-1.870)\end{array}$ & 0.253 \\
\hline $\begin{array}{c}\text { Recessive } \\
(\mathrm{CC}+\mathrm{CT} \text { vs. TT) } \\
\text { HWE-P }\end{array}$ & & & $\begin{array}{c}0.713 \\
(0.256-1.988)\end{array}$ & 0.518 & & & $\begin{array}{c}1.233 \\
(0.452-3.364)\end{array}$ & 0.682 & & & $\begin{array}{c}1.451 \\
(0.583-3.611)\end{array}$ & 0.424 \\
\hline
\end{tabular}

Note: AOR was adjusted by age, gender, hypertension, and diabetes mellitus. AOR = adjusted odds ratio; 95\% CI = 95\% confidence interval; HWE = Hardy-Weinberg equilibrium. 


\subsection{Haplotype Analysis of Polymorphisms with Synergistic Effects}

To investigate the genes in the absence of environmental influence, the combined effects of HOTAIR alleles and prevalence of CAD were analyzed (Table 4). Our analysis indicated that there were significant effects resulting from combined genes (rs1899663 $\mathrm{G}>\mathrm{T} / \mathrm{rs} 12826786 \mathrm{C}>\mathrm{T}$ ) when environmental influence was excluded from CAD risk in the analysis of two SNPs (T-T: OR, 1.448, 95\% CI, 1.128-1.859). In our analysis of three SNPs, rs1899663 G > T/rs12826786 C > T/rs874945 C >T and rs1899663 G>T / rs920778 T>C/rs12826786 $\mathrm{C}>\mathrm{T}$ were significantly different between the $\mathrm{CAD}$ and control groups (T-T-C: OR, 1.517, 95\% CI, 1.176-1.956; T-C-T: OR, 1.493, 95\% CI, 1.151-1.936). The haplotype A-T (rs4759314 $\mathrm{A}>\mathrm{G}$ rs1899663 G>T) was also found to be significantly associated with CAD risk (A-T: OR, $1.289,95 \% \mathrm{CI}, 1.021-1.628)$. When haplotypes, such as A-T-T (rs4759314 A>G/rs1899663 $\mathrm{G}>\mathrm{T} / \mathrm{rs} 12826786 \mathrm{C}>\mathrm{T} ; p=0.008), \mathrm{A}-\mathrm{T}-\mathrm{T}-\mathrm{T}(\mathrm{rs} 4759314 \mathrm{~A}>\mathrm{G} / \mathrm{rs} 1899663 \mathrm{G}>\mathrm{T} / \mathrm{rs} 12826786$ $\mathrm{C}>\mathrm{T} / \mathrm{rs} 874945 \mathrm{C}>\mathrm{T} ; p=0.0003$ ), and A-T-C-T-T (rs4759314 A>G/rs1899663 G>T/rs920778 $\mathrm{T}>\mathrm{C} / \mathrm{rs} 12826786 \mathrm{C}>\mathrm{T} / \mathrm{rs} 874945 \mathrm{C}>\mathrm{T} ; p<0.0001$ ), were combined, a significant difference between CAD patients and healthy controls was observed (A-T-T: OR, 1.405, 95\% CI, 1.0921.807; A-T-T-T: OR, 1.474, 95\% CI, 1.141-1.904; A-T-C-T-T: OR, 1.815, 95\% CI, 1.396-2.359).

Table 4. Haplotype analysis of HOTAIR polymorphisms in CAD and controls subjects.

\begin{tabular}{|c|c|c|c|c|}
\hline Haplotype & $\begin{array}{c}\text { Controls } \\
(2 n=836)\end{array}$ & $\begin{array}{c}\text { Case } \\
(2 n=884)\end{array}$ & OR $(95 \% \mathrm{CI})$ & $p$-Value \\
\hline \multicolumn{5}{|c|}{ rs4759314 A>G/ rs1899663 G>T/ rs920778 T>C/ rs7958904 G>C/ rs12826786 C>T/ rs874945 C>T } \\
\hline A-G-T-G-C-C & 0.6855 & 0.7014 & 1.000 (reference) & \\
\hline A-G-T-G-C-T & 0.0137 & 0.0012 & $0.084(0.011-0.653)$ & 0.003 \\
\hline A-G-T-C-C-C & 0.0109 & 0.0022 & $0.205(0.044-0.955)$ & 0.033 \\
\hline A-G-T-C-T-T & 0.0062 & 0.0000 & $0.084(0.005-1.524)$ & 0.026 \\
\hline A-T-C-C-T-C & 0.0081 & 0.0000 & $0.062(0.004-1.082)$ & 0.006 \\
\hline A-T-C-C-T-T & 0.1197 & 0.1909 & $1.562(1.190-2.050)$ & 0.001 \\
\hline G-G-T-G-C-C & 0.0088 & 0.0000 & $0.062(0.004-1.082)$ & 0.006 \\
\hline \multicolumn{5}{|c|}{ rs4759314 A>G/ rs1899663 G>T/ rs920778 T>C/ rs7958904 G>C/ rs12826786 C>T } \\
\hline A-G-T-G-C & 0.6992 & 0.7025 & 1.000 (reference) & \\
\hline A-G-T-C-C & 0.0121 & 0.0022 & $0.188(0.041-0.864)$ & 0.019 \\
\hline A-G-T-C-T & 0.0064 & 0.0000 & $0.086(0.005-1.553)$ & 0.027 \\
\hline A-T-C-C-T & 0.1269 & 0.1909 & $1.502(1.149-1.963)$ & 0.003 \\
\hline G-G-T-G-C & 0.0098 & 0.0000 & $0.055(0.003-0.963)$ & 0.003 \\
\hline \multicolumn{5}{|c|}{$\mathrm{rs} 4759314 \mathrm{~A}>\mathrm{G} / \mathrm{rs} 1899663 \mathrm{G}>\mathrm{T} / \mathrm{rs} 920778 \mathrm{~T}>\mathrm{C} / \mathrm{rs} 12826786 \mathrm{C}>\mathrm{T} / \mathrm{rs} 874945 \mathrm{C}>\mathrm{T}$} \\
\hline A-G-T-C-C & 0.6949 & 0.7034 & 1.000 (reference) & \\
\hline A-G-T-C-T & 0.0149 & 0.0012 & $0.091(0.012-0.704)$ & 0.004 \\
\hline A-G-T-T-T & 0.0095 & 0.0000 & $0.064(0.004-1.119)$ & 0.008 \\
\hline A-T-C-T-C & 0.0094 & 0.0000 & $0.064(0.004-1.119)$ & 0.008 \\
\hline A-T-C-T-T & 0.1287 & 0.2023 & $1.815(1.396-2.359)$ & $<0.0001$ \\
\hline G-G-T-C-C & 0.0100 & 0.0012 & $0.137(0.017-1.098)$ & 0.041 \\
\hline \multicolumn{5}{|c|}{ rs4759314 A>G/ rs1899663 G>T/rs7958904 G>C/ rs12826786 C>T/ rs874945 C>T } \\
\hline A-G-G-C-C & 0.6923 & 0.7045 & 1.000 (reference) & \\
\hline A-G-G-C-T & 0.0136 & 0.0025 & $0.169(0.037-0.766)$ & 0.011 \\
\hline A-G-C-T-T & 0.0160 & 0.0046 & $0.286(0.093-0.882)$ & 0.026 \\
\hline A-T-C-T-C & 0.0094 & 0.0000 & $0.055(0.003-0.950)$ & 0.003 \\
\hline A-T-C-T-T & 0.1275 & 0.1955 & $1.503(1.151-1.961)$ & 0.003 \\
\hline G-G-G-C-C & 0.0122 & 0.0023 & $0.186(0.041-0.852)$ & 0.019 \\
\hline \multicolumn{5}{|c|}{ rs4759314 A>G/ rs1899663 G>T/ rs920778 T>C/ rs12826786 C>T } \\
\hline A-G-T-C & 0.7088 & 0.7047 & 1.000 (reference) & \\
\hline A-G-T-T & 0.0097 & 0.0000 & $0.056(0.003-0.973)$ & 0.003 \\
\hline A-T-C-T & 0.1378 & 0.2022 & $1.482(1.142-1.921)$ & 0.003 \\
\hline G-G-T-C & 0.0112 & 0.0012 & $0.106(0.013-0.838)$ & 0.010 \\
\hline \multicolumn{5}{|c|}{ rs4759314 A>G/ rs1899663 G>T/ rs7958904 G>C/ rs12826786 C>T } \\
\hline A-G-G-C & 0.7055 & 0.7068 & 1.000 (reference) & \\
\hline A-G-C-T & 0.0167 & 0.0046 & $0.270(0.088-0.824)$ & 0.017 \\
\hline A-T-C-T & 0.1365 & 0.1954 & $1.433(1.102-1.861)$ & 0.007 \\
\hline G-G-G-C & 0.0132 & 0.0036 & $0.258(0.071-0.928)$ & 0.031 \\
\hline
\end{tabular}


Table 4. Cont.

\begin{tabular}{|c|c|c|c|c|}
\hline Haplotype & $\begin{array}{l}\text { Controls } \\
(2 n=836)\end{array}$ & $\begin{array}{c}\text { Case } \\
(2 n=884)\end{array}$ & OR $(95 \%$ CI $)$ & $p$-Value \\
\hline \multicolumn{5}{|c|}{ rs4759314 A>G/ rs1899663 G>T/ rs12826786 C>T/ rs874945 C>T } \\
\hline A-G-C-C & 0.7050 & 0.7108 & 1.000 (reference) & \\
\hline A-G-C-T & 0.0149 & 0.0024 & $0.156(0.035-0.702)$ & 0.006 \\
\hline A-G-T-T & 0.0216 & 0.0046 & $0.208(0.070-0.620)$ & 0.002 \\
\hline A-T-T-C & 0.0099 & 0.0000 & $0.055(0.003-0.959)$ & 0.003 \\
\hline A-T-T-T & 0.1425 & 0.2114 & $1.474(1.141-1.904)$ & 0.003 \\
\hline \multicolumn{5}{|c|}{ rs4759314 A>G/ rs1899663 G>T/ rs12826786 C>T } \\
\hline A-G-C & 0.7187 & 0.7131 & 1.000 (reference) & \\
\hline A-G-T & 0.0221 & 0.0047 & $0.212(0.071-0.630)$ & 0.002 \\
\hline A-T-T & 0.1522 & 0.2114 & $1.405(1.092-1.807)$ & 0.008 \\
\hline G-G-C & 0.0675 & 0.0424 & $0.630(0.410-0.969)$ & 0.034 \\
\hline \multicolumn{5}{|c|}{ rs1899663 G>T/ rs12826786 C>T } \\
\hline $\mathrm{G}-\mathrm{C}$ & 0.7861 & 0.7555 & 1.000 (reference) & \\
\hline G-T & 0.0225 & 0.0047 & $0.207(0.070-0.612)$ & 0.002 \\
\hline $\mathrm{T}-\mathrm{T}$ & 0.1522 & 0.2114 & $1.448(1.128-1.859)$ & 0.004 \\
\hline
\end{tabular}

$\mathrm{CAD}$, coronary artery disease; $\mathrm{OR}$, odds ratio; $\mathrm{CI}$, confidence interval.

\subsection{Interaction Analysis between Genes and Clinical Factors}

We analyzed the possibility of synergistic effects between genes and clinical factors such as metabolic syndrome, DM, hypertension, smoking, folate, and homocysteine (Table 5). Our results showed that rs1899663 has a synergistic effect between DM and metabolic syndrome ( $\mathrm{rs} 1899663 \mathrm{GT}+\mathrm{TT} / \mathrm{DM}$, AOR $=3.276,95 \% \mathrm{CI}=1.786-6.009 ; \mathrm{rs} 1899663$ $\mathrm{GT}+\mathrm{TT} /$ metabolic syndrome, $\mathrm{AOR}=5.389,95 \% \mathrm{CI}=3.272-8.877)$. When HOTAIR rs 12826786 $\mathrm{CC}+\mathrm{TT}$ was combined with hypertension $(\mathrm{AOR}=2.012,95 \% \mathrm{CI}=1.325-3.057), \mathrm{DM}(\mathrm{AOR}$ $=2.755,95 \% \mathrm{CI}=1.527-4.968)$, or metabolic syndrome $(\mathrm{AOR}=5.364,95 \% \mathrm{CI}=3.244-8.870)$, we observed an increased association with CAD. Combining the rs920778 TC+CC type with hypertension $(\mathrm{AOR}=1.895,95 \% \mathrm{CI}=1.274-2.818), \mathrm{DM}(\mathrm{AOR}=2.757,95 \% \mathrm{CI}=1.604-4.738)$, and metabolic syndrome ( $\mathrm{AOR}=5.811,95 \% \mathrm{CI}=3.496-9.660)$ resulted in an increased prevalence towards CAD risk. Figure 1 shows that rs 1899663 had the highest synergistic effect with hypertension, metabolic syndrome, and DM, while rs12826786 had the greatest synergy with DM, metabolic syndrome, hypertension, and folate.

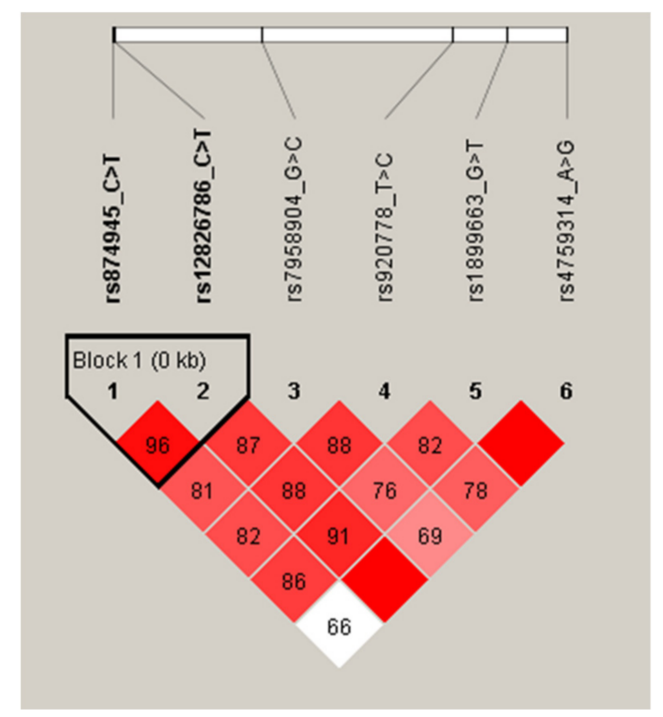

Figure 1. LD patterns of HOTAIR gene polymorphisms in all participants. 
Table 5. Interaction analysis between genotype and patients characteristics in participants.

\begin{tabular}{|c|c|c|c|c|c|c|c|c|}
\hline Characteristics & rs1899663 GG & $p$ & rs1899663 GG+GT & $p$ & rs920778 TT & $p$ & rs920778 TT+TC & $p$ \\
\hline \multicolumn{9}{|l|}{ Hypertension } \\
\hline No & 1.000 (reference) & & $1.230(0.834-1.814)$ & 0.296 & 1.000 (reference) & & $1.217(0.833-1.779)$ & 0.310 \\
\hline Yes & $1.424(0.989-2.049)$ & 0.057 & 1.967 (1.302-2.972) & 0.001 & $1.514(1.029-2.227)$ & 0.036 & 1.895 (1.274-2.818) & 0.002 \\
\hline \multicolumn{9}{|l|}{$\begin{array}{l}\text { Diabetes } \\
\text { mellitus }\end{array}$} \\
\hline No & 1.000 (reference) & & $1.275(0.934-1.742)$ & 0.126 & 1.000 (reference) & & $1.236(0.911-1.679)$ & 0.174 \\
\hline Yes & $1.969(1.262-3.071)$ & 0.003 & $3.276(1.786-6.009)$ & 0.000 & $2.110(1.308-3.405)$ & 0.002 & $2.757(1.604-4.738)$ & 0.000 \\
\hline \multicolumn{9}{|l|}{ Smoking } \\
\hline No & 1.000 (reference) & & $1.276(0.902-1.805)$ & 0.169 & 1.000 (reference) & & $1.234(0.880-1.731)$ & 0.224 \\
\hline \multirow{2}{*}{\multicolumn{9}{|c|}{$\begin{array}{l}\text { Metabolic } \\
\text { syndrome }\end{array}$}} \\
\hline & & & & & & & & \\
\hline No & 1.000 (reference) & & $1.533(1.040-2.262)$ & 0.031 & 1.000 (reference) & & $1.283(0.874-1.882)$ & 0.203 \\
\hline Yes & $4.898(3.169-7.570)$ & $<0.0001$ & $5.389(3.272-8.877)$ & $<0.0001$ & $3.989(2.536-6.275)$ & $<0.0001$ & $5.811(3.496-9.660)$ & $<0.0001$ \\
\hline \multicolumn{9}{|c|}{ 6. } \\
\hline$<25 \mathrm{~kg} / \mathrm{m}^{2}$ & 1.000 (reference) & & $1.591(1.108-2.284)$ & 0.012 & 1.000 (reference) & & $1.356(0.951-1.933)$ & 0.092 \\
\hline$\geq 25 \mathrm{~kg} / \mathrm{m}^{2}$ & $3.900(2.646-5.749)$ & $<0.0001$ & $3.479(2.243-5.394)$ & $<0.0001$ & $3.588(2.375-5.420)$ & $<0.0001$ & $3.560(2.331-5.438)$ & $<0.0001$ \\
\hline \multicolumn{9}{|l|}{ Triglycerides } \\
\hline$<150 \mathrm{mg} / \mathrm{dL}$ & 1.000 (reference) & & $0.955(0.667-1.365)$ & 0.799 & 1.000 (reference) & & $0.949(0.668-1.349)$ & 0.772 \\
\hline$\geq \underset{\text { Folate }}{\geq 150 \mathrm{mg} / \mathrm{dL}}$ & $0.949(0.661-1.364)$ & 0.778 & $2.223(1.423-3.473)$ & 0.000 & $0.927(0.632-1.361)$ & 0.700 & $1.844(1.210-2.810)$ & 0.004 \\
\hline$>4.01 \mathrm{nmol} / \mathrm{L}$ & 1.000 (reference) & & $1.381(1.016-1.877)$ & 0.039 & 1.000 (reference) & & $1.251(0.928-1.688)$ & 0.142 \\
\hline$\leq 4.01 \mathrm{nmol} / \mathrm{L}$ & $2.343(1.323-4.151)$ & 0.004 & $2.109(1.165-3.820)$ & 0.014 & $2.142(1.153-3.978)$ & 0.016 & $2.097(1.198-3.669)$ & 0.010 \\
\hline Characteristics & rs7958904 GG & $p$ & rs7958904 GG+GC & $p$ & rs12826786 CC & $p$ & rs12826786 CC+CT & $p$ \\
\hline \multicolumn{9}{|l|}{ Hypertension } \\
\hline No & 1.000 (reference) & & $1.135(0.774-1.665)$ & 0.516 & 1.000 (reference) & & $1.376(0.928-2.041)$ & 0.113 \\
\hline Yes & $1.506(1.031-2.202)$ & 0.034 & $1.797(1.201-2.688)$ & 0.004 & $1.512(1.057-2.163)$ & 0.024 & $2.012(1.325-3.057)$ & 0.001 \\
\hline \multicolumn{9}{|l|}{$\begin{array}{l}\text { Diabetes } \\
\text { mellitus }\end{array}$} \\
\hline No & 1.000 (reference) & & $1.197(0.880-1.630)$ & 0.252 & 1.000 (reference) & & $1.392(1.014-1.910)$ & 0.041 \\
\hline Yes & $2.307(1.428-3.727)$ & 0.001 & $2.300(1.353-3.910)$ & 0.002 & $2.188(1.403-3.410)$ & 0.001 & $2.755(1.527-4.968)$ & 0.001 \\
\hline \multicolumn{9}{|l|}{ Smoking } \\
\hline No & 1.000 (reference) & & $1.226(0.872-1.724)$ & 0.242 & 1.000 (reference) & & $1.357(0.954-1.931)$ & 0.089 \\
\hline Yes & $0.801(0.505-1.270)$ & 0.345 & $0.913(0.556-1.499)$ & 0.720 & $0.750(0.484-1.164)$ & 0.200 & $1.108(0.664-1.848)$ & 0.695 \\
\hline \multicolumn{9}{|l|}{$\begin{array}{l}\text { Metabolic } \\
\text { syndrome }\end{array}$} \\
\hline No & 1.000 (reference) & & $1.338(0.911-1.966)$ & 0.138 & 1.000 (reference) & & $1.539(1.038-2.281)$ & 0.032 \\
\hline Yes & $4.296(2.769-6.664)$ & $<0.0001$ & 5.745 (3.399-9.709) & $<0.0001$ & $4.464(2.921-6.823)$ & $<0.0001$ & $5.364(3.244-8.870)$ & $<0.0001$ \\
\hline \multicolumn{9}{|c|}{ 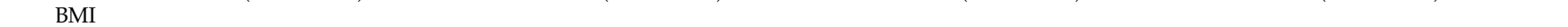 } \\
\hline$<25 \mathrm{~kg} / \mathrm{m}^{2}$ & 1.000 (reference) & & $1.287(0.902-1.836)$ & 0.164 & 1.000 (reference) & & $1.616(1.120-2.331)$ & 0.010 \\
\hline$\geq 25 \mathrm{~kg} / \mathrm{m}^{2}$ & $3.485(2.339-5.192)$ & $<0.0001$ & $3.503(2.258-5.436)$ & $<0.0001$ & $3.779(2.581-5.535)$ & $<0.0001$ & $3.477(2.228-5.426)$ & $<0.0001$ \\
\hline \multicolumn{9}{|l|}{ Triglycerides } \\
\hline$<150 \mathrm{mg} / \mathrm{dL}$ & 1.000 (reference) & & $0.962(0.674-1.373)$ & 0.830 & 1.000 (reference) & & $1.026(0.712-1.478)$ & 0.890 \\
\hline $\begin{array}{c}\geq 150 \mathrm{mg} / \mathrm{dL} \\
+ \text { Folate }\end{array}$ & $1.027(0.703-1.499)$ & 0.892 & $1.609(1.063-2.436)$ & 0.025 & $1.003(0.700-1.436)$ & 0.989 & $2.129(1.368-3.314)$ & 0.001 \\
\hline$>4.01 \mathrm{nmol} / \mathrm{L}$ & 1.000 (reference) & & $1.184(0.877-1.600)$ & 0.271 & 1.000 (reference) & & $1.369(1.004-1.868)$ & 0.048 \\
\hline$\leq 4.01 \mathrm{nmol} / \mathrm{L}$ & $2.268(1.246-4.129)$ & 0.007 & $1.897(1.065-3.379)$ & 0.030 & $2.056(1.185-3.567)$ & 0.010 & $2.360(1.263-4.408)$ & 0.007 \\
\hline
\end{tabular}

CAD, coronary artery disease; AOR, adjusted odds ratio; $95 \% \mathrm{CI}, 95 \%$ confidence interval. The adjusted odds ratio on the basis of risk factors, such as age, gender, hypertension, and diabetes mellitus. + Folate $4.01 \mathrm{nmol} / \mathrm{L}$ is lower than the $15 \%$ cut-off for each level in CAD patients and controls.

\subsection{Clinical Variables in Ischemic Stroke Patients Stratified by HOTAIR Polymorphism Status}

We conducted a variance analysis between the genes and clinical factors in all participants and subgroups (Table 6). In all participants, the HDL cholesterol level was found to be significantly associated with HOTAIR polymorphisms, including rs920778 T $>$ C, rs 12826786 $\mathrm{C}>\mathrm{T}$, and rs874945 C>T ( $p=0.040,0.049$, and 0.031, respectively). The TG level demonstrated a decreasing trend depending on the $\operatorname{rs} 1899663 \mathrm{G}>\mathrm{T}$ polymorphism $(p=0.31)$. In addition, rs12826786 C $>\mathrm{T}$ and rs874945 $\mathrm{C}>\mathrm{T}$ exhibited a decreasing trend depending on the polymorphisms in the control group (Table 7). In contrast, rs4759314 A>G showed an increasing trend depending on the polymorphisms in the control group. In the CAD group, the TG level was significantly associated with $\mathrm{rs} 920778 \mathrm{~T}>\mathrm{C}(p=0.037)$, while the HDL cholesterol level was associated with $\operatorname{rs} 12826786 \mathrm{C}>\mathrm{T}(p=0.023)$ and $\mathrm{rs} 874945 \mathrm{C}>\mathrm{T}$ $(p=0.029)$ (Table 8). Finally, our data demonstrated that rs920778 T>C and rs7958904 G>C displayed a decreasing trend depending on the polymorphisms. 
Table 6. Clinical variables in CAD patients and controls stratified by HOTAIR polymorphisms status by ANOVA.

\begin{tabular}{|c|c|c|c|c|c|c|c|c|c|c|c|}
\hline \multirow[t]{2}{*}{ Genotypes } & $\begin{array}{c}\text { BMI } \\
\left(\mathrm{kg} / \mathrm{m}^{2}\right)\end{array}$ & $\begin{array}{c}\text { Total } \\
\text { Cholesterol } \\
\text { (mg/dL) }\end{array}$ & $\begin{array}{l}\text { Triglyceride } \\
\text { (mg/dL) }\end{array}$ & $\begin{array}{c}\text { HDL } \\
\text { Cholsterol } \\
\text { (mg/dL) }\end{array}$ & $\begin{array}{c}\text { LDL } \\
\text { Cholesterol } \\
\text { (mg/dL) }\end{array}$ & $\begin{array}{c}\text { FBS } \\
(\mathrm{mg} / \mathrm{dL})\end{array}$ & $\begin{array}{c}\text { SBP } \\
(\mathrm{mmHg})\end{array}$ & $\underset{(\mathrm{mmHg})}{\mathrm{DBP}}$ & $\begin{array}{c}\text { Folate } \\
(\mathrm{mg} / \mathrm{mL})\end{array}$ & $\underset{(\mathrm{pg} / \mathrm{mL})}{\text { Vitamin B12 }}$ & $\begin{array}{l}\text { Homocysteine } \\
(\mathrm{mmol} / \mathrm{L})\end{array}$ \\
\hline & $\begin{array}{l}\text { Mean } \pm \text { SD } \\
\quad(703)\end{array}$ & $\begin{array}{c}\text { Mean } \pm \text { SD } \\
\quad(846)\end{array}$ & $\begin{array}{c}\text { Mean } \pm \text { SD } \\
\quad(846)\end{array}$ & $\begin{array}{c}\text { Mean } \pm \text { SD } \\
(616)\end{array}$ & $\begin{array}{c}\text { Mean } \pm \text { SD } \\
\quad(602)\end{array}$ & $\begin{array}{c}\text { Mean } \pm \text { SD } \\
\quad(838)\end{array}$ & $\begin{array}{c}\text { Mean } \pm \text { SD } \\
\quad(860)\end{array}$ & $\begin{array}{c}\text { Mean } \pm S D \\
\mathbf{( 8 6 0 )}\end{array}$ & $\begin{array}{l}\text { Mean } \pm \text { SD } \\
\quad(817)\end{array}$ & Mean \pm SD (502) & $\begin{array}{l}\text { Mean } \pm \text { SD } \\
\quad(836)\end{array}$ \\
\hline \multicolumn{12}{|c|}{ rs4759314 A>G } \\
\hline AA & $24.82 \pm 3.41$ & $189.65 \pm 43.00$ & $152.63 \pm 92.05$ & $44.64 \pm 12.03$ & $113.60 \pm 41.47$ & $127.39 \pm 53.30$ & $129.91 \pm 18.97$ & $79.85 \pm 12.27$ & $8.77 \pm 8.38$ & $689.81 \pm 269.93$ & $9.71 \pm 4.35$ \\
\hline $\mathrm{AG}$ & $24.70 \pm 3.11$ & $188.89 \pm 38.66$ & $152.11 \pm 82.23$ & $42.99 \pm 12.36$ & $113.28 \pm 38.67$ & $132.68 \pm 58.30$ & $126.62 \pm 20.15$ & $76.81 \pm 12.87$ & $8.80 \pm 6.28$ & $673.49 \pm 317.61$ & $10.02 \pm 4.98$ \\
\hline GG & $24.93 \pm 3.83$ & $218.33 \pm 74.00$ & $208.67 \pm 86.23$ & & & $95.33 \pm 2.08$ & $128.33 \pm 16.07$ & $78.33 \pm 15.28$ & $9.29 \pm 5.27$ & $613.00 \pm 191.27$ & $10.45 \pm 3.98$ \\
\hline$p$-value & 0.953 & 0.501 & 0.567 & 0.291 & 0.952 & 0.395 & 0.296 & 0.084 & 0.993 & 0.818 & 0.789 \\
\hline GG & $24.65 \pm 3.39$ & $189.04 \pm 40.11$ & $151.70 \pm 90.66$ & $43.85 \pm 11.33$ & $113.91 \pm 40.65$ & $128.36 \pm 54.41$ & $130.00 \pm 19.50$ & $79.59 \pm 12.65$ & $8.69 \pm 6.82$ & $682.64 \pm 258.63$ & $9.68 \pm 4.36$ \\
\hline GT & $25.07 \pm 3.41$ & $192.02 \pm 45.02$ & $154.67 \pm 91.12$ & $45.46 \pm 13.60$ & $113.10 \pm 40.52$ & $124.10 \pm 50.44$ & $129.19 \pm 18.22$ & $79.54 \pm 11.96$ & $9.20 \pm 10.42$ & $689.76 \pm 300.19$ & $9.90 \pm 4.55$ \\
\hline TT & $25.01 \pm 3.00$ & $180.58 \pm 55.82$ & $153.24 \pm 97.16$ & $44.77 \pm 9.07$ & $112.89 \pm 50.21$ & $149.67 \pm 65.10$ & $126.32 \pm 20.22$ & $78.50 \pm 11.70$ & $6.55 \pm 4.18$ & $768.43 \pm 349.37$ & $9.35 \pm 4.18$ \\
\hline$p$-value & 0.296 & 0.066 & 0.906 & 0.297 & 0.970 & 0.019 & 0.476 & 0.871 & 0.190 & 0.518 & 0.681 \\
\hline \multicolumn{12}{|c|}{ rs920778 T>C } \\
\hline TT & $24.66 \pm 3.33$ & $189.08 \pm 40.95$ & $151.48 \pm 90.00$ & $43.37 \pm 10.75$ & $114.91 \pm 41.76$ & $127.62 \pm 53.97$ & $130.12 \pm 19.38$ & $79.78 \pm 12.48$ & $8.69 \pm 6.79$ & $609.68 \pm 254.33$ & $9.56 \pm 4.21$ \\
\hline $\mathrm{TC}$ & $24.98 \pm 3.54$ & $191.49 \pm 45.31$ & $155.89 \pm 93.60$ & $45.94 \pm 13.55$ & $113.46 \pm 41.99$ & $127.53 \pm 53.50$ & $129.22 \pm 18.67$ & $79.60 \pm 12.42$ & $9.09 \pm 10.16$ & $685.32 \pm 280.53$ & $9.94 \pm 4.57$ \\
\hline $\mathrm{CC}$ & $25.06 \pm 2.54$ & $183.35 \pm 40.27$ & $144.69 \pm 84.10$ & $44.36 \pm 11.93$ & $103.09 \pm 28.13$ & $131.78 \pm 54.62$ & $126.57 \pm 19.17$ & $76.69 \pm 10.60$ & $7.56 \pm 4.96$ & $696.29 \pm 273.63$ & $10.15 \pm 5.24$ \\
\hline \multicolumn{12}{|c|}{ rs7958904 G>C } \\
\hline GG & $24.73 \pm 3.38$ & $188.83 \pm 41.54$ & $150.37 \pm 88.44$ & $43.36 \pm 10.76$ & $115.25 \pm 41.65$ & $127.35 \pm 53.73$ & $129.79 \pm 19.61$ & $79.41 \pm 12.60$ & $8.80 \pm 6.83$ & $669.91 \pm 275.67$ & $9.52 \pm 4.09$ \\
\hline $\mathrm{CC}$ & $25.37 \pm 3.26$ & $184.00 \pm 38.90$ & $149.17 \pm 91.64$ & $45.00 \pm 11.43$ & $107.17 \pm 30.85$ & $132.47 \pm 51.46$ & $130.95 \pm 23.12$ & $78.81 \pm 13.24$ & $7.53 \pm 4.85$ & $697.38 \pm 276.20$ & $10.04 \pm 5.40$ \\
\hline$p$-value & 0.466 & 0.330 & 0.546 & 0.095 & 0.397 & 0.793 & 0.693 & 0.802 & 0.477 & 0.621 & 0.327 \\
\hline \multicolumn{12}{|c|}{ rs $12826786 C>T$} \\
\hline $\mathrm{CC}$ & $24.65 \pm 3.41$ & $188.63 \pm 40.16$ & $150.74 \pm 90.27$ & $43.56 \pm 11.15$ & $113.88 \pm 40.22$ & $128.08 \pm 54.68$ & $129.71 \pm 19.63$ & $79.33 \pm 12.52$ & $8.69 \pm 6.75$ & $683.65 \pm 267.04$ & $9.68 \pm 4.29$ \\
\hline $\mathrm{CT}$ & $25.05 \pm 3.36$ & $193.24 \pm 47.50$ & $157.98 \pm 94.52$ & $46.10 \pm 13.66$ & $114.77 \pm 44.08$ & $127.00 \pm 52.48$ & $129.30 \pm 18.30$ & $80.05 \pm 12.26$ & $9.16 \pm 10.58$ & $698.35 \pm 292.93$ & $9.88 \pm 4.63$ \\
\hline TT & $25.52 \pm 2.58$ & $172.04 \pm 31.84$ & $138.28 \pm 61.58$ & $44.39 \pm 9.56$ & $97.18 \pm 22.10$ & $132.00 \pm 50.08$ & $129.20 \pm 16.63$ & $77.84 \pm 9.96$ & $6.30 \pm 4.06$ & $643.20 \pm 286.57$ & $9.60 \pm 4.68$ \\
\hline$p$-value & 0.205 & 0.069 & 0.403 & 0.049 & 0.166 & 0.895 & 0.954 & 0.576 & 0.230 & 0.756 & 0.822 \\
\hline \multicolumn{12}{|c|}{ rs874945 C>T } \\
\hline $\mathrm{CC}$ & $24.68 \pm 3.43$ & $188.97 \pm 39.82$ & $150.22 \pm 90.50$ & $43.49 \pm 11.23$ & $114.64 \pm 40.21$ & $128.35 \pm 54.99$ & $129.91 \pm 19.75$ & $79.56 \pm 12.58$ & $8.58 \pm 6.70$ & $683.42 \pm 267.15$ & $9.71 \pm 4.46$ \\
\hline $\mathrm{CT}$ & $25.01 \pm 3.37$ & $193.02 \pm 47.76$ & $158.11 \pm 93.62$ & $46.19 \pm 13.43$ & $113.63 \pm 44.35$ & $125.22 \pm 50.56$ & $129.07 \pm 18.22$ & $79.74 \pm 12.31$ & $9.37 \pm 10.58$ & $693.50 \pm 286.89$ & $9.84 \pm 4.39$ \\
\hline TT & $25.02 \pm 2.73$ & $173.66 \pm 34.78$ & $146.95 \pm 77.00$ & $44.01 \pm 10.51$ & $100.30 \pm 24.21$ & $141.03 \pm 60.20$ & $128.42 \pm 16.73$ & $77.45 \pm 9.52$ & $6.86 \pm 4.52$ & $702.25 \pm 320.37$ & $9.51 \pm 4.14$ \\
\hline$p$-value & 0.437 & 0.040 & 0.459 & 0.031 & 0.188 & 0.228 & 0.776 & 0.559 & 0.064 & 0.905 & 0.875 \\
\hline
\end{tabular}

ANOVA, analysis of variance; HDL cholesterol, high-density lipoprotein cholesterol; LDL cholesterol, low-density lipoprotein cholesterol; BMI, body mass index; SD, standard deviation. 
Table 7. Clinical variables in healthy controls stratified by HOTAIR polymorphisms status by ANOVA.

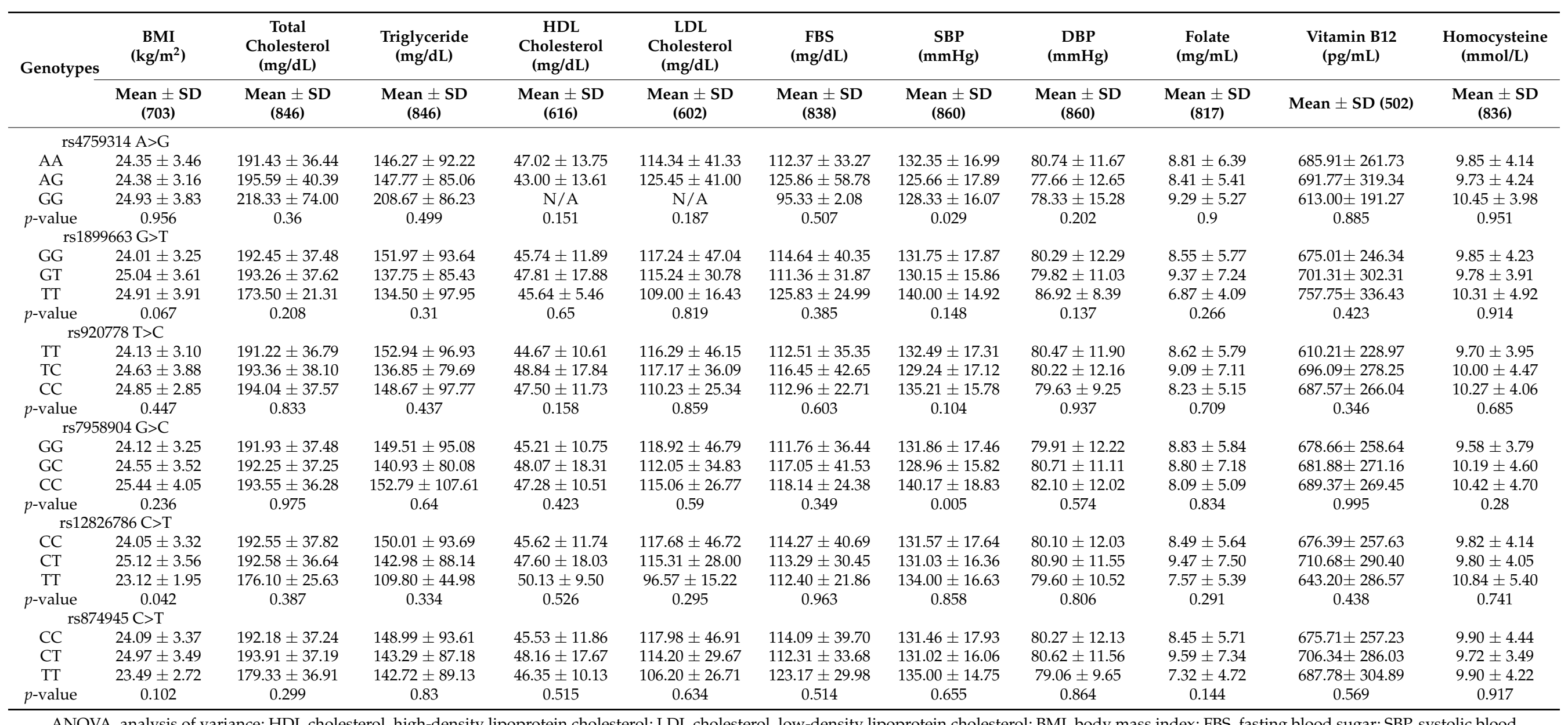

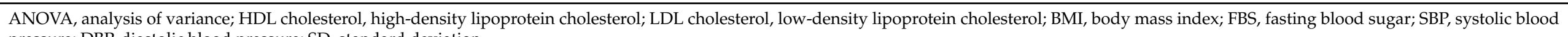
pressure; DBP, diastolic blood pressure; SD, standard deviation. 
Table 8. Clinical variables in CAD patients stratified by HOTAIR polymorphisms status by ANOVA.

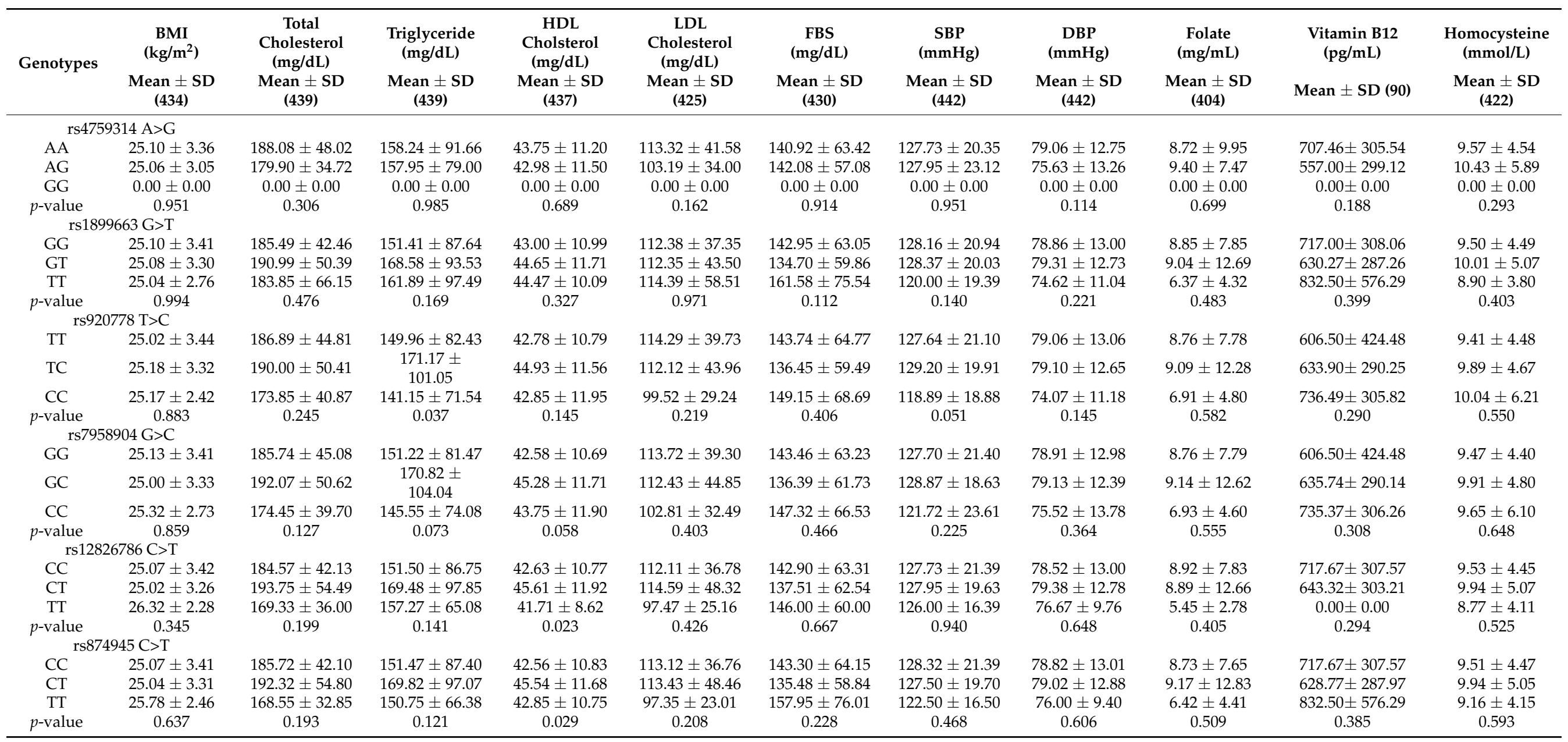

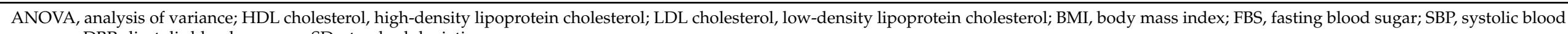
pressure; DBP, diastolic blood pressure; SD, standard deviation. 


\section{Discussion}

In this study, we investigated the association between CAD occurrence and the lncRNA HOTAIR. Our results show that the allelic frequencies of the rs1899663 and rs7958904 SNPs were significantly different between the control and CAD groups in genotype frequencies. These variants have previously been reported in association with other diseases, including various cancers, sclerosis, and psychiatric conditions [7,19,23,24]. Amongst metabolic syndrome patients, rs1899663 and rs12826786 were found to be associated with CAD risk. In our haplotype analysis, rs1899663 G>T and rs12826786 C>T (T-T) were significantly associated with CAD risk. Most of the other haplotypes were significantly associated with protective responses in CAD. These results demonstrate that HOTAIR plays a role in reducing the occurrence of $\mathrm{CAD}$. Further studies are needed to investigate the underlying mechanism of how HOTAIR regulates CAD.

HOTAIR, which is approximately $2.2 \mathrm{~kb}$ and consists of six exons, is located between HOXC11 and HOXC12 on chromosome 12. Studies have shown that the $5^{\prime}$-end of HOTAIR binds PRC2 and the $3^{\prime}$-end binds LSD1, leading to epigenetic changes and transcriptional regulation [9]. At first, HOTAIR was reported as a cancer-related gene [6,23,25]; however, further investigation revealed that HOTAIR is associated with other conditions as well, including pregnancy, psoriasis, and CAD $[5,26,27]$. HOTAIR overexpression leads to decreased interleukin (IL)-17, IL-23, and tumor necrosis factor alpha, as well as inhibition of nuclear factor kappa B activation in lipopolysaccharide-treated chondrocytes [28].

CAD has been associated with endothelial dysfunction, in that endothelial lncRNA influences heart disease prevalence [12,29,30]. HIF1A-AS2 and APO1 expression levels were found to be significantly increased in CAD patients [29]. HOTAIR was revealed to be associated with various heart diseases in vivo. It was reported that overexpressed HOTAIR also affects the receptor of advanced glycation end-products (RAGE) that promoted inflammatory responses in an AMI modeling rat [31]. Additionally, HOTAIR was found to regulate oxidative stress, which could progress ischemia-reperfusion (I/R) injury in myocardial model mice [32]. HOTAIR is also significantly different between CAD and non-CAD patients. As mentioned, IncRNA functionally associates with microRNAs [12], preventing them from binding target genes. HOTAIR inactivates miR-1, which is involved in acute myocardial infarction (AMI) and sponging miR-613 to regulate Connexin 43 in atrial fibrillation, suggesting a protective role for HOTAIR in this disease [11,33].

The coronary artery is a blood vessel that supplies oxygen to the heart muscle. CAD results from the blockage of blood flow, which ultimately leads to a lack of oxygen and nutrients to the heart. CAD is caused by several environmental factors, including hyperlipidemia, alcohol, smoking, hypertension, and genetic risk factors [34]. The heritability of CAD risk factors has been reported to increase the occurrence of CAD [35]. Genes reported to be associated with CAD risk are usually related to environmental factors, such as the LDL receptor, apolipoprotein B, and protein convertase subtilisin/kexin type 9. A few reports have suggested that HOTAIR is associated with blood vessels or CAD. For example, HOTAIR expression was shown to be significantly increased in cardiomyocytes in the septic mouse model [36]. Another study reported that HOTAIR was significantly downregulated in the early phase of acute myocardial infarction [33].

Dysfunction of lncRNA is associated with disrupted gene expression and chromatin inactivation [34,35]. Recently, lncRNAs have been proposed as potential biomarkers. The lncRNA autophagy-promoting factor (APF) regulates $A T G 7$ via miR-188-3p, leading to the regulation of MI [37]. Previous reports demonstrated that HOTAIR overexpression is associated with miR-19, suggesting its role as a potential marker in hypertrophy [36]. Moreover, in vivo studies revealed that miR-519d-3p is associated with MI because of the sponging effect of HOTAIR [16]. Combining HOTAIR with other lncRNAs that have been associated with CAD, such as APO1, HIF1A-AS2, and GAS5, may constitute a novel diagnostic and therapeutic strategy for treating CAD [29,38].This study has several limitations. First, our sample sizes were small and must be verified in a future study utilizing a larger sample population. However, our results were statistically significant. Second, our study 
population was limited to Korean individuals. Additional investigations including other ethnic groups may reveal different patterns; however, the genotypes of each polymorphism examined must be confirmed to be in HWE. Third, our study was based only on blood samples. The genotypes and allelic frequencies must be confirmed in tissue samples from the placenta or endometrium to exclude the possibility of genetic mosaicism. The effects of HOTAIR SNPs should also be confirmed using in vitro studies. Fourth, we analyzed CAD patients and a control group without any history of CAD; however we did not analyze all risk factors such as coagulation factors. For these limitations, we conducted statistical analyses and adjusted for risk factors, including age, gender, hyperlipidemia, diabetes, and hypertension, which affect CAD prevalence, to reduce differences between CAD patients and the control group. In our study population, although there was no significant difference, the LDL level of patients was lower than control group and folate levels were significantly higher in the patients group. As these are unordinary results, they could not exclude that patients may have an additional regimen or nutritional supplement to improve folate and LDL levels.

\section{Conclusions}

We demonstrated that the HOTAIR rs4759314 A>G, rs1899663 C>T, and rs12826786 $\mathrm{C}>\mathrm{T}$ polymorphisms are genetically associated with $\mathrm{CAD}$ in the Korean population. The rs1899663 and rs12826786 polymorphisms were associated with increased CAD risk, but rs4759314 was linked to a decreased CAD risk. This study suggests that the HOTAIR polymorphisms may contribute to $\mathrm{CAD}$ and may be potential biomarkers for assessing $\mathrm{CAD}$ risk. Further studies using larger cohorts are required to better understand the role of HOTAIR as a biomarker. Moreover, in vivo and vitro studies are necessary to learn which function of HOTAIR influences downstream gene expression and ultimately leads to CAD. This knowledge would help in CAD diagnosis and could ultimately lead to the development of effective therapeutic strategies.

Supplementary Materials: The following are available online at https:/ /www.mdpi.com/article/10 .3390/jpm11050375/s1, Table S1: Details of HOTAIR polymorphisms for PCR-RFLP analysis.

Author Contributions: I.-J.K. and J.-Y.L. conceived of and designed the experiments. H.-W.P. analyzed data. H.-S.P. and E.-J.K. conducted the experiments. J.-H.S. and N.-K.K. supervised the manuscript writing. All authors have read and agreed to the published version of the manuscript.

Funding: This work was supported by the National Research Foundation of Korea (NRF) grant funded by the Korean government (MSIT) (2017R1D1A1B03030110 and 2018R1D1A1A09082764).

Institutional Review Board Statement: The study was approved by the Institutional Review Board of CHA Bundang Medical Center. (IRB number: 2013-10-114).

Informed Consent Statement: Not applicable.

Data Availability Statement: Data sharing not applicable.

Conflicts of Interest: The authors declare no conflict of interest.

\section{References}

1. Cam, S.F.; Sekuri, C.; Tengiz, I.; Ercan, E.; Sagcan, A.; Akin, M.; Berdeli, A. The G894T polymorphism on endothelial nitric oxide synthase gene is associated with premature coronary artery disease in a Turkish population. Thromb. Res. 2005, 116, 287-292. [CrossRef] [PubMed]

2. Chaer, R.A.; Billeh, R.; Massad, M.G. Genetics and gene manipulation therapy of premature coronary artery disease. Cardiology 2004, 101, 122-130. [CrossRef] [PubMed]

3. Visvikis-Siest, S.; Marteau, J.-B. Genetic variants predisposing to cardiovascular disease. Curr. Opin. Lipidol. 2006, 17, 139-151. [CrossRef] [PubMed]

4. Yarmishyn, A.A.; Kurochkin, I. V Long noncoding RNAs: A potential novel class of cancer biomarkers. Front. Genet. 2015, 6, 145. [CrossRef]

5. Avazpour, N.; Hajjari, M.; Yazdankhah, S.; Sahni, A.; Foroughmand, A.M. Circulating HOTAIR RNA is potentially up-regulated in coronary artery disease. Genom. Inform. 2018, 16, e25. [CrossRef] 
6. Wu, L.; Zhang, L.; Zheng, S. Role of the long non-coding RNA HOTAIR in hepatocellular carcinoma. Oncol. Lett. 2017, 14, 1233-1239. [CrossRef]

7. Li, J.; Liu, R.; Tang, S.; Feng, F.; Wang, X.; Qi, L.; Liu, C.; Yao, Y.; Sun, C. The effect of long noncoding RNAs HOX transcript antisense intergenic RNA single-nucleotide polymorphisms on breast cancer, cervical cancer, and ovarian cancer susceptibility: A meta-analysis. J. Cell. Biochem. 2019, 120, 7056-7067. [CrossRef]

8. Zheng, D.; Hou, Y.; Li, Y.; Bian, Y.; Khan, M.; Li, F.; Huang, L.; Qiao, C. Long Non-coding RNA Gas5 Is Associated with Preeclampsia and Regulates Biological Behaviors of Trophoblast via MicroRNA-21. Front. Genet. 2020, 11, 188. [CrossRef]

9. Tang, Q.; Hann, S.S. HOTAIR: An oncogenic long non-coding RNA in human cancer. Cell. Physiol. Biochem. 2018, 47, 893-913. [CrossRef]

10. Greco, S.; Zaccagnini, G.; Perfetti, A.; Fuschi, P.; Valaperta, R.; Voellenkle, C.; Castelvecchio, S.; Gaetano, C.; Finato, N.; Beltrami, A.P.; et al. Long noncoding RNA dysregulation in ischemic heart failure. J. Transl. Med. 2016, 14, 183. [CrossRef]

11. Dai, W.; Chao, X.; Li, S.; Zhou, S.; Zhong, G.; Jiang, Z. Long Noncoding RNA HOTAIR Functions as a Competitive Endogenous RNA to Regulate Connexin43 Remodeling in Atrial Fibrillation by Sponging MicroRNA-613. Cardiovasc. Ther. 2020, 2020, 5925342. [CrossRef]

12. Charles, S.; Natarajan, J. Two way network construction and analysis of mRNA, miRNA and lncRNA reveals critical regulators and regulatory modules in cardiovascular diseases. Genes Genom. 2020, 42, 855-867. [CrossRef]

13. Bhan, A.; Mandal, S.S. LncRNA HOTAIR: A master regulator of chromatin dynamics and cancer. Biochim. Biophys. Acta 2015, 1856, 151-164. [CrossRef]

14. Milevskiy, M.J.G.; Al-Ejeh, F.; Saunus, J.M.; Northwood, K.S.; Bailey, P.J.; Betts, J.A.; McCart Reed, A.E.; Nephew, K.P.; Stone, A.; Gee, J.M.W.; et al. Long-range regulators of the lncRNA HOTAIR enhance its prognostic potential in breast cancer. Hum. Mol. Genet. 2016, 25, 3269-3283. [CrossRef]

15. Peng, Y.; Meng, K.; Jiang, L.; Zhong, Y.; Yang, Y.; Lan, Y.; Zeng, Q.; Cheng, L. Thymic stromal lymphopoietin-induced HOTAIR activation promotes endothelial cell proliferation and migration in atherosclerosis. Biosci. Rep. 2017, 37. [CrossRef]

16. Zhang, D.; Wang, B.; Ma, M.; Yu, K.; Zhang, Q.; Zhang, X. lncRNA HOTAIR Protects Myocardial Infarction Rat by Sponging miR-519d-3p. J. Cardiovasc. Transl. Res. 2019, 12, 171-183. [CrossRef]

17. Zhou, H.; Wang, B.; Yang, Y.; Jia, Q.; Zhang, A.; Qi, Z.; Zhang, J. Long Noncoding RNAs in Pathological Cardiac Remodeling: A Review of the Update Literature. Biomed. Res. Int. 2019, 2019, 7159592. [CrossRef]

18. Lin, Y.; Ge, Y.; Wang, Y.; Ma, G.; Wang, X.; Liu, H.; Wang, M.; Zhang, Z.; Chu, H. The association of rs710886 in lncRNA PCAT1 with bladder cancer risk in a Chinese population. Gene 2017, 627, 226-232. [CrossRef]

19. Sayad, A.; Badrlou, E.; Ghafouri-Fard, S.; Taheri, M. Association Analysis Between the rs1899663 Polymorphism of HOTAIR and Risk of Psychiatric Conditions in an Iranian Population. J. Mol. Neurosci. 2020, 70, 953-958. [CrossRef]

20. MacArthur DG, T.-S.C. Loss-of-function variants in the genomes of healthy humans. Hum. Mol. Genet. 2010, 19, R125-R130. [CrossRef]

21. Ritchie, M.D.; Hahn, L.W.; Roodi, N.; Bailey, L.R.; Dupont, W.D.; Parl, F.F.; Moore, J.H. Multifactor-dimensionality reduction reveals high-order interactions among estrogen-metabolism genes in sporadic breast cancer. Am. J. Hum. Genet. 2001, 69, 138-147. [CrossRef] [PubMed]

22. Hahn, L.W.; Ritchie, M.D.; Moore, J.H. Multifactor dimensionality reduction software for detecting gene-gene and geneenvironment interactions. Bioinformatics 2003, 19, 376-382. [CrossRef] [PubMed]

23. Kim, J.O.; Jun, H.H.; Kim, E.J.; Lee, J.Y.; Park, H.S.; Ryu, C.S.; Kim, S.; Oh, D.; Kim, J.W.; Kim, N.K. Genetic Variants of HOTAIR Associated with Colorectal Cancer Susceptibility and Mortality. Front. Oncol. 2020, 10, 72. [CrossRef] [PubMed]

24. Taheri, M.; Noroozi, R.; Sadeghpour, S.; Omrani, M.D.; Ghafouri-Fard, S. The rs4759314 SNP within Hotair lncRNA is associated with risk of multiple sclerosis. Mult. Scler. Relat. Disord. 2020, 40, 101986. [CrossRef]

25. Ren, K.; Li, Y.; Lu, H.; Li, Z.; Li, Z.; Wu, K.; Li, Z.; Han, X. Long Noncoding RNA HOTAIR Controls Cell Cycle by Functioning as a Competing Endogenous RNA in Esophageal Squamous Cell Carcinoma. Transl. Oncol. 2016, 9, 489-497. [CrossRef]

26. Rakhshan, A.; Zarrinpour, N.; Moradi, A.; Ahadi, M.; Omrani, M.D.; Ghafouri-Fard, S.; Taheri, M. A single nucleotide polymorphism within HOX Transcript Antisense RNA (HOTAIR) is associated with risk of psoriasis. Int. J. Immunogenet. 2020, 47, 430-434. [CrossRef]

27. Mohammadpour-Gharehbagh, A.; Jahantigh, D.; Saravani, M.; Harati-Sadegh, M.; Maruie-Milan, R.; Teimoori, B.; Salimi, S. Impact of HOTAIR variants on preeclampsia susceptibility based on blood and placenta and in silico analysis. IUBMB Life 2019, 71, 1367-1381. [CrossRef]

28. Haemmig, S.; Simion, V.; Feinberg, M.W. Long Non-Coding RNAs in Vascular Inflammation. Front. Cardiovasc. Med. 2018, 5, 22. [CrossRef]

29. Videira, R.F.; da Costa Martins, P.A.; Falcão-Pires, I. Non-Coding RNAs as Blood-Based Biomarkers in Cardiovascular Disease. Int. J. Mol. Sci. 2020, 21, 9285. [CrossRef]

30. Tan, W.L.; Xu, M.; Liu, Z.; Wu, T.Y.; Yang, Y.; Luo, J.; Yang, J.; Luo, Y. HOTAIR inhibited intracellular Ca ${ }^{2+}$ via regulation of Cav1.2 channel in human cardiomyocytes. Cell. Mol. Biol. 2015, 61, 79-83.

31. Lu, W.; Zhu, L.; Ruan, Z.-B.; Wang, M.-X.; Ren, Y.; Li, W. HOTAIR promotes inflammatory response after acute myocardium infarction by upregulating RAGE. Eur. Rev. Med. Pharmacol. Sci. 2018, 22, 7423-7430. [CrossRef] 
32. Meng, K.; Jiao, J.; Zhu, R.-R.; Wang, B.-Y.; Mao, X.-B.; Zhong, Y.-C.; Zhu, Z.-F.; Yu, K.-W.; Ding, Y.; Xu, W.-B.; et al. The Long Noncoding RNA Hotair Regulates Oxidative Stress and Cardiac Myocyte Apoptosis during Ischemia-Reperfusion Injury. Oxid. Med. Cell. Longev. 2020, 2020, 1645249. [CrossRef]

33. Gao, L.; Liu, Y.; Guo, S.; Yao, R.; Wu, L.; Xiao, L.; Wang, Z.; Liu, Y.; Zhang, Y. Circulating Long Noncoding RNA HOTAIR is an Essential Mediator of Acute Myocardial Infarction. Cell. Physiol. Biochem. 2017, 44, 1497-1508. [CrossRef]

34. Malakar, A.K.; Choudhury, D.; Halder, B.; Paul, P.; Uddin, A.; Chakraborty, S. A review on coronary artery disease, its risk factors, and therapeutics. J. Cell. Physiol. 2019, 234, 16812-16823. [CrossRef]

35. Nora, J.J.; Lortscher, R.H.; Spangler, R.D.; Nora, A.H.; Kimberling, W.J. Genetic-epidemiologic study of early-onset ischemic heart disease. Circulation 1980, 61, 503-508. [CrossRef]

36. Wu, H.; Liu, J.; Li, W.; Liu, G.; Li, Z. LncRNA-HOTAIR promotes TNF- $\alpha$ production in cardiomyocytes of LPS-induced sepsis mice by activating NF-кB pathway. Biochem. Biophys. Res. Commun. 2016, 471, 240-246. [CrossRef]

37. Wang, K.; Liu, C.-Y.; Zhou, L.-Y.; Wang, J.-X.; Wang, M.; Zhao, B.; Zhao, W.-K.; Xu, S.-J.; Fan, L.-H.; Zhang, X.-J.; et al. APF lncRNA regulates autophagy and myocardial infarction by targeting miR-188-3p. Nat. Commun. 2015, 6, 6779. [CrossRef]

38. Yin, Q.; Wu, A.; Liu, M. Plasma Long Non-Coding RNA (lncRNA) GAS5 is a New Biomarker for Coronary Artery Disease. Med. Sci. Monit. 2017, 23, 6042-6048. [CrossRef] 